\title{
EL PAPEL DE LA KATHARSIS EN EL PROGRAMA ARISTOTÉLICO DE EDUCACIÓN MUSICAL. SU RELACIÓN CON LA FUNCIÓN LÚDICA Y CON LA EDUCATIVA DE LA MOUSIKE
}

\author{
Viviana Suñol 1 \\ Instituto de Investigaciones en Humanidades y Ciencias Sociales, Universidad \\ Nacional de La Plata - CONICET (Argentina)
}

Recibido: 17.09.2018 - Aceptado: 30.10.2018

\section{RESUMEN}

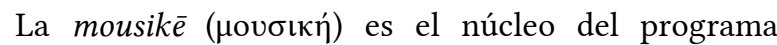
educativo que Aristóteles diseña en la Política para el mejor régimen. El filósofo le asigna a esta disciplina diferentes funciones y propone una consideración jerárquica de ellas. Aunque menciona explícitamente tres propósitos musicales, a saber, el juego, la educación y el ocio, en el último capítulo de la obra refiere a

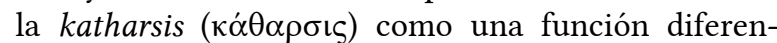
ciada, cuyo vínculo con las restantes no precisa (Pol. 1341b38-40). Mi propósito en el presente trabajo es mostrar que resulta problemático determinar el lugar que ocupa la katharsis en el esquema de las funciones musicales que Aristóteles bosqueja en Pol. VIII 3, 5-7, en especial, en relación con la función lúdica y con la educativa. En tal sentido, intentaré demostrar que su posición oscila entre ambas y que, en el caso particular de los ciudadanos del mejor régimen, la función ética es condición de la katharsis. Con vistas a ello, analizaré los pasajes de Pol. VIII 5-7 en los que el filósofo tácita o explícitamente refiere a este término, con especial atención a la valiosa y singular consideración general sobre su significado, que ofrece en Pol. 1342a5-15.

Palabras clave: katharsis; emociones; mousikē; política; educación; ética.

\begin{abstract}

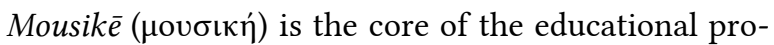
gram that Aristotle designs in Politics for the best regime. The philosopher assigns to this discipline different functions and proposes a hierarchical consideration of them. Although he explicitly mentions three musical purposes, namely, play, education and leisure, in the last chapter of the work refers to katharsis ( $\kappa \dot{\alpha} \theta \alpha \rho \sigma \iota \varsigma$ ) as a differentiated function, whose relation with the others does not precise (Pol. 1341b38-40). My purpose in this paper is to show that it is problematic to determine the place that katharsis occupies in the scheme of musical functions that Aristotle draws in Pol. 8. 3, 5-7, especially in relation to the playful and the educational functions. In this sense, I will try to demonstrate that its position oscillates between them and that, in the particular case of the citizens of the best regime, the ethical function is a condition of $\mathrm{ka}$ tharsis. To achieve this purpose, I will analyze the passages in Pol. 8. 5-7 in which the philosopher tacit or explicitly refers to katharsis, with special attention to the valuable and singular general consideration of its meaning that he presents in Pol. 1342a5-15.
\end{abstract}

Keywords: katharsis; emotions; mousikē; politics; education; ethics.

\footnotetext{
${ }^{1}$ vsunyol@gmail.com
} 


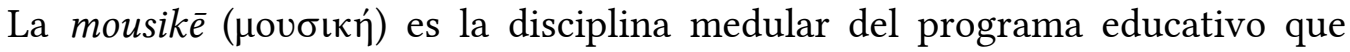
Aristóteles diseña para el mejor régimen en Política VIII 3-7. ${ }^{2}$ El filósofo le asigna diferentes funciones y propone una consideración jerárquica de ellas, conforme a la cual, la función más elevada, es decir, la ociosa, está esencial-

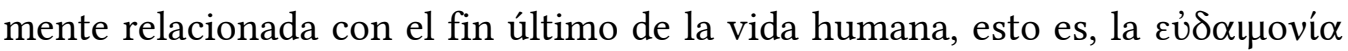
(Pol. 1338a1-3). A lo largo de Pol. VIII, el Estagirita menciona explícitamente tres propósitos musicales, a saber, el juego, la educación y el ocio (Pol. 1339a14-26; b12-14; 1341b40). Sin embargo, en Pol. VIII 7 (1341b38-41) presenta a la katharsis

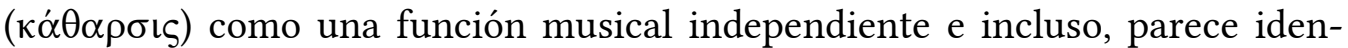
tificar al ocio con la relajación. ${ }^{3}$ Este pasaje plantea varias dificultades para el estudio de cuáles y cuántas son las funciones musicales y cómo se vinculan entre sí algunas de ellas. Estas cuestiones podrían ser consideradas secundarias en el contexto general de su filosofía; de hecho, el propio Aristóteles reconoce el carácter esquemático de su análisis sobre las armonías y los ritmos para la educación, para el cual se apoya en una división que no es de su autoría e incluso delega a otros especialistas la investigación exacta de este aspecto puntual. Quizás este papel, en apariencia subsidiario, sea la razón por la cual, las funciones musicales prácticamente no han sido objeto de estudio sistemático por parte de la literatura académica. ${ }^{4}$ No obstante, se trata de un asunto estrechamente vinculado a los cimientos de su pensamiento ético-político, lo cual se manifiesta en las correspondencias que estos guardan con el currículo musical. En efecto, el papel, la estructura curricular y las funciones que le otorga a la mousikē son piezas claves para comprender las respuestas a las dos preguntas fundamentales que el filósofo plantea al comienzo de Pol. VII, a saber, cuál es el

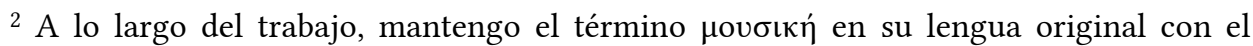
propósito de evidenciar la distancia conceptual que existe entre nuestro empleo actual de la palabra "música" y la amplia noción griega, la cual era constitutiva de su identidad (Rocconi 2015, 82). Asimismo, al mantener el vocablo griego procuro destacar el hecho de que también resulta problemático establecer qué es lo que Aristóteles entiende por este término en Pol. VII 3, 5-7, lo cual es objeto de debate entre los intérpretes del texto. Al respecto, véase n. 8 .

${ }^{3}$ Puesto que uno de los objetivos del presente trabajo es dilucidar el significado de la

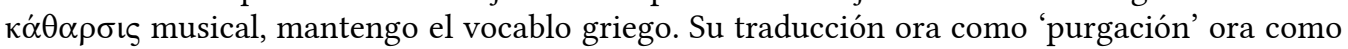
'purificación' nos sumerge en el inabarcable y aún vigente debate sobre su significado. Precisamente, mi lectura subraya el hecho de que el Estagirita nos ofrece una consideración general sobre cómo entiende este término en Pol. VIII 7 (1342a5-15). Sobre la controversia acerca de "la preocupación distractiva" acerca de cómo traducir el término, cfr. Halliwell 1986, 198.

${ }^{4} \mathrm{Si}$ bien estos problemas aparecen tratados en las distintas ediciones, traducciones y comentarios del texto de Pol. VIII, no es posible encontrar artículos específicamente dedicados al estudio de las funciones musicales. Empero, constituyen una excepción los trabajos recientes de Pierre Destrée 2017 y 2018, cuyo interés se centra en la función ociosa y, en particular, en la compleja alusión a esta en Pol. VIII 7 (1341b40). 
mejor régimen y cuál es la vida mejor (Pol. 1323a 14-16). Por un lado, sus restricciones sobre la ejecución musical en la vida adulta de los ciudadanos se corresponden con la alternancia generacional como modelo de organización política del mejor régimen. ${ }^{5}$ Por otra parte, la relación entre la función ética y la ociosa de la mousikē permite elucidar el vínculo entre la vida política y la vida filosófica y, en virtud de ello, resulta indispensable para comprender el ßíos

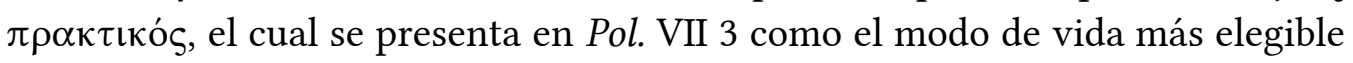

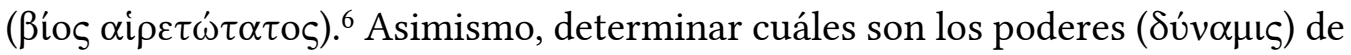
la mousikē y cómo estos se relacionan entre sí, permite entender de manera concreta en qué consiste el ideal ético-político aristotélico, al esclarecer los distintos aspectos que conforman la vida cultural de los ciudadanos del mejor régimen.

En el presente trabajo me focalizo en el papel que Aristóteles le otorga a la katharsis en la jerarquía de las funciones musicales que bosqueja en Pol. VIII 3 , 5-7, en especial, en relación con la función lúdica y con la educativa. Mi propósito aquí es determinar cuál es el lugar que ocupa en dicha jerarquía: si tal como sugiere el pasaje de Pol. VIII 7- constituye una función independiente de las otras tres que el filósofo menciona o si, como proponen algunos intérpretes, debe ser identificada con la relajación y, por ende, se correspondería con la función lúdica de la mousikē, siguiendo el esquema tripartito de funciones. El análisis del papel que juega la katharsis no solo permite comprender el proceso emocional que la mousikē pone en juego, sino también reflexionar sobre la relación que las experiencias emocionales, en general, tienen con la educación de los ciudadanos del mejor régimen. Con vistas a esclarecer algunas de estas cuestiones, y teniendo en cuenta la promesa incumplida de Aristóteles de explicar qué entiende por katharsis en sus escritos sobre poética, intentaré dilucidar el rol que cumple dicha función, atendiendo a cómo describe su significado "de manera general" ( $\dot{\alpha} \pi \lambda \tilde{\omega} \varsigma$ ) en Pol. VIII 7 y a lo que se desprende de su empleo del término en Pol. VIII 6 (1341a21-24), a propósito de la prohibición del uso del aulós e incluso considerando su implícita alusión a ella

\footnotetext{
${ }^{5}$ La alternancia generacional como modelo de organización es condición para lograr que la dominación sea política, esto es, entre iguales y, a su vez, garantiza la estabilidad del mejor régimen. Aunque Aristóteles no hace ninguna referencia explícita, dicha alternancia se corresponde, desde el punto de vista musical, con las restricciones sobre la ejecución que establece para los ciudadanos adultos del mejor régimen, a quienes les está vedado participar de las mismas, limitándolas a los jóvenes. Para un análisis más detallado de esta cuestión y de la posible objeción a esta correspondencia, cfr. Suñol 2017, 11-17.

${ }^{6}$ En este caso, la correspondencia es más compleja de identificar y de explicar. Muy brevemente puede consignarse que tanto la mejor forma de vida como la función musical más alta se caracterizan por su carácter no instrumental y reflexivo, el cual es propio de la actividad teórica. Sin embargo, en ambos casos lo práctico, esto es, la vida política y el empleo ético de la

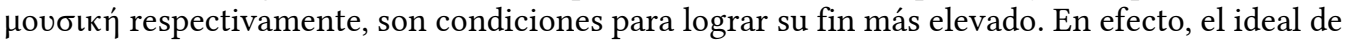
la vida más elegible es un modo de vida teórica que presupone la vida política, así también la función ociosa de la $\mu$ ovбıкń implica el aprendizaje musical y requiere haber sido habituado en la virtud mediante la ejecución, es decir, la función ética. Cfr. Suñol 2017, 18-32.
} 
en Pol. VIII 5 (1340a7-12), mediante la referencia al entusiasmo que producen las melodías de Olimpo.

\section{1}

A diferencia de la literatura académica que usualmente recurre al texto de Pol. VIII para esclarecer el significado de katharsis en la definición de tragedia que Aristóteles ofrece en Poética 6 (1449b24-28), el punto de partida de esta lectura es el estudio de las funciones musicales en Pol. y, en particular, el lugar problemático que la katharsis ocupa, el cual, quizás, podría eventualmente ayudar a esclarecer su enigmático empleo en Poet. Más allá de la referencia explícita que el propio filósofo hace en Pol. VIII 7 (1341b38-40) a su definición del término en sus escritos sobre poética, ${ }^{7}$ la katharsis plantea una innegable tensión en relación a ambos textos. La consideración casi subsidiaria de la katharsis musical que, en principio, se desprende de Pol. VIII se contrapone con la importancia que el término tiene en Poet. En efecto, si esta es una mera forma de relajación y, por ende, estaría vedada para los ciudadanos del mejor régimen, entonces cómo es posible que el filósofo la considere en Poet. como el fin último del que -a su juicio- es el género poético por excelencia. Si bien la conexión entre ambas obras es un tema complejo, cuyo estudio excede los límites del presente trabajo, ${ }^{8}$ baste señalar aquí que el propósito de Aristóteles en cada una de ellas es distinto, por lo cual difiere su perspectiva sobre el arte mimético. ${ }^{9} \mathrm{En}$ correspondencia con ello, nuestro interés por la katharsis no es instrumental, es decir, no es con vistas a elucidar su empleo en la Poet., sino que surge de la indagación sobre la relevancia filosófica del programa de educación musical propuesto en Pol. VIII 3, 5-7 y, en particular, del estudio de sus funciones.

En Pol. VIII 5 Aristóteles menciona explícitamente los tres propósitos de la mousikē (Pol. 1339b13-14), los cuales ya había descrito en Pol. VIII 3, cuando analiza las razones que motivaron a los antiguos a la inclusión de esta disciplina en el esquema educativo tradicional y allí es donde con más énfasis defiende el carácter ocioso de la mousike $e^{10}$ Precisamente, lo que la distingue de las restantes disciplinas es que no se emplea con vistas a ninguna necesidad ni utilidad, en

${ }^{7}$ Sobre el significado de esta referencia, véase p. 131.

${ }^{8}$ Existe una amplia discusión académica sobre la posibilidad de establecer vínculos entre

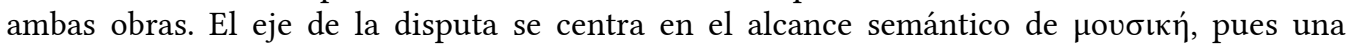
comprensión amplia del mismo permite conectar Poet. y Pol. VIII (Lord 1982). Sin embargo, una comprensión estrecha, como la que propone Ford 2004, 314-315, impide otorgar un valor educacional a la tragedia, reduciendo su función a un mero entretenimiento.

${ }^{9}$ En Pol. VIII su interés se centra en la educación de los ciudadanos del mejor régimen, por lo cual sus consideraciones musicales están supeditadas a su propósito ético-político, mientras que en Poet. adopta una perspectiva que, en términos modernos, podríamos denominar propiamente estética. Sobre esta cuestión, cfr. Destrée 2011, 18-20.

10 Sobre la noción de ocio y la singularidad de su empleo en Aristóteles, véase Nightingale 1996, 30-39. 
virtud de lo cual encarna el modelo de la educación liberal y ociosa (Pol. 1338a30-32). ${ }^{11}$ El hecho de que se trata de una actividad que no tiene otro propósito más allá de sí misma es lo que determina la superioridad de este empleo, pues favorece el desarrollo del intelecto. En efecto, la función ociosa de la mousiké permite que los futuros ciudadanos del mejor régimen ejerzan su discernimiento, esto es, su capacidad de juicio; para ello es preciso que previamente participen de un estricto programa de educación musical que se inicia en la infancia y que, probablemente, se prolonga a lo largo de toda la vida. ${ }^{12}$ El uso ocioso de la mousikē conlleva un aprendizaje de carácter no instrumental (Pol. 1338a10), que tácitamente la emparienta con la filosofía, si bien Aristóteles no hace ninguna referencia explícita a dicha conexión. ${ }^{13}$ Aunque resulte sorprendente, en los restantes capítulos de Pol. VIII le presta escasa atención a la función ociosa, ${ }^{14}$ pues su interés primariamente pedagógico determina que el eje de su análisis sea su propósito ético, el cual permite que los jóvenes adquieran la virtud y sean capaces de juzgar éticamente la mousikē (Pol. 1339b3-4). ${ }^{15}$ En varias ocasiones, Aristóteles destaca la raigambre antropológica del placer musical (Pol. 1339b20-22); de ahí su referencia al "placer común" del que todos los hombres de todas las edades y caracteres tienen sensación y que afecta incluso a otros animales, esclavos y niños (Pol. 1340a2-5; 1341a15-18). El placer acompaña a todas y cada una de las funciones musicales, en razón de ello es posible establecer una tácita jerarquía conforme a la cual, el placer concomitante al ejercicio de la función ociosa constituye su forma más elevada (Pol. 1339b17-19). El placer musical es también una de las principales razones que permiten que sea un instrumento pedagógico privilegiado en la habituación ética de los jóvenes (Pol. 1340b15-19). La forma más elemental del placer musical refiere a su empleo lúdico, el cual corresponde al descanso de las tensiones del trabajo (Pol. 1339b26-40). Mientras que las funciones ociosas y éticas de la mousikè solo pueden ser empleadas por los ciudadanos del mejor régimen, su

${ }^{11}$ A pesar de la importancia que le reconoce, Aristóteles no solo no afirma que esta sea la única actividad ociosa a la que deben dedicarse los ciudadanos, sino que incluso se pregunta en Pol. VIII 3 (1338a32-33) si la educación liberal de los hijos comprende una o más disciplinas de esta clase, cuestión que deja sin resolver. Cfr. Kraut 1997, 183 y Destrée 2013, 316 n. 15.

${ }^{12}$ No hay acuerdo en la literatura sobre si la educación musical se prolonga en la vida adulta, p. ej. Lord 1982, 34, y Ford 2004, 326 n. 5. Cabe recordar que el final de Pol. VIII 7 (1342b27-29) revela que las prescripciones sobre el empleo de las armonías se prolongan hasta la vejez de los ciudadanos. Para una síntesis de las distintas interpretaciones sobre la educación en la vida adulta, cfr. Ford 2004, 328 n. 61.

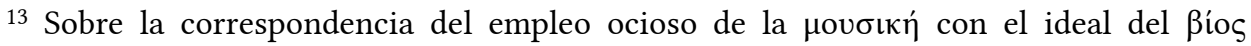

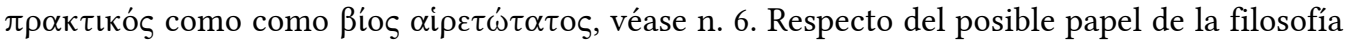
en el programa educativo del mejor régimen, Cfr. Suñol 2015, 67 n.54.

${ }^{14}$ En Pol. VIII 5 (1339a25-26) se limita a mencionar a la función ociosa y, como veremos a continuación, en Pol. VIII 7 parece identificarla con el descanso, lo cual contradice sus consideraciones sobre la singularidad de esta disciplina expuestas en Pol. VIII 3. Sobre esta cuestión me ocuparé con mayor detenimiento más adelante en el trabajo. Véanse notas 24 y 25.

${ }^{15}$ Sobre la distinción entre el discernimiento ético al que refiere en Pol. VIII 5 y el juicio estético en Pol. VIII 6, cfr. Suñol 2017, 16-17. 
utilización para el juego y el descanso abarca a los trabajadores. Aristóteles no especifica si los ciudadanos pueden emplear la mousikē con fines lúdicos y de relajación, pero -como veremos a propósito de la katharsis-, algunos indicios abonan esta posibilidad. A pesar de que el ocio es el fin último de la vida de los ciudadanos del mejor régimen, estos no están exentos de experimentar las tensiones que necesariamente conlleva la vida cívica y, por ende, es muy probable que Aristóteles admitiera la posibilidad de que estos también emplearan la mousikē con vistas a la relajación. ${ }^{16}$ Tal como sugieren sus prescripciones sobre la ejecución musical, las limitaciones que establece sobre el empleo de las melodías entusiásticas y sus consideraciones sobre la censura es posible que instituyera restricciones respecto al empleo de este tipo de mousikē por parte de los ciudadanos.

Aristóteles no hace ninguna referencia explícita a la katharsis en Pol. VIII 5 , pero sin dudas resulta significativo el hecho de que apele a las melodías de Olimpo para ejemplificar la influencia ética de la mousikē en el carácter, las cuales -asegura- producen entusiasmo en el alma (Pol. 1340a8-12). ${ }^{17}$ La referencia a este músico legendario de la Frigia arcaica se condice con las consideraciones etnomusicológicas que el filósofo hace a lo largo de Pol. VIII (1341a20-24, b6-8;1342b1-7), a saber, la censura del aulós, entre los instrumentos, del frigio, entre las armonías y del ditirambo, en la poesía, es decir, de todo aquello que propicie el descontrol emocional y que lleve a los hombres a estar fuera de sí. ${ }^{18}$ Tal como revelan las prescripciones aristotélicas, el carácter extático de la mousike era tradicionalmente asociado a todo aquello que fuera de origen frigio. ${ }^{19}$ Hemos visto que la función ética de la mousike tiene como propósito que los futuros ciudadanos del mejor régimen logren la moderación emocional habituándolos a amar y odiar correctamente, gracias a su naturaleza simpatética

${ }^{16}$ Destrée 2011, 22, se apoya en el pasaje de Pol. VIII 3 (1337b33-1338a1) para respaldar su idea de que los ciudadanos tienen necesidad de vez en cuando de descansar después de un esfuerzo y que emplean la música para ello. A mi entender, el pasaje en cuestión es netamente aristocrático y establece una tácita escisión entre los ciudadanos ociosos y los trabajadores excluidos de la ciudadanía. Sin embargo, un análisis cabal de la vida de los ciudadanos del mejor régimen revela la necesidad del descanso por parte de estos. Más adelante en el trabajo analizaré los otros indicios que, a mi entender, abonan esta idea.

17 Aristóteles refiere reiteradamente a esta emoción en Pol. VIII (1340a10-12; 1341b34; 1342a4,7), pero no la menciona ni en Retórica ni en Ética a Nicómaco. Literalmente, el entusiasmo alude al estar poseído por un dios, lo cual significa que el individuo se encuentra en un estado de trance o frenesí, provocado por el contacto directo con el ser divino. Si bien en Pol. VIII 7 refiere a los cantos sagrados, su empleo del término parece aludir más bien al estar fuera de sí, i.e. a la excitación emocional. Cfr. Kraut 1997, 193.

18 Platón en el Banquete 265c hace referencia a las melodías de Olimpo, cuya autoría atribuye al mítico músico Marsias y destaca el encantamiento que las mismas producían por medio de la flauta.

${ }^{19}$ Rocconi 2010, 43, advierte sobre las limitaciones que, de manera general, supone una aproximación etnomusicológica en la reconstrucción de la comprensión de las antiguas fuentes musicales griegas. 
y placentera, lo cual etno-musicalmente se corresponde con el dorio. ${ }^{20}$ Pero, paradójicamente, recurre al desborde que producen las melodías entusiásticas para ilustrar no solo la influencia emocional de la mousikē, sino su función ética. ${ }^{21}$ En virtud de lo cual, podría decirse que, en este punto de la obra, la función catártica de la mousikē parece ser parte o estar subsumida en su función ética. ${ }^{22}$

\section{2}

En el último capítulo de Pol., Aristóteles presenta una enumeración de las funciones musicales que se contrapone en varios aspectos con la división tripartita inicial.

Decimos que es preciso emplear la mousikē no con vistas a una sola utilidad, sino para muchas (no solo en virtud de la educación y de

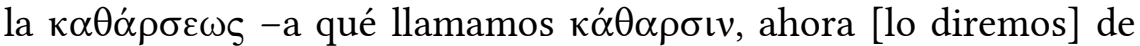
manera general $(\alpha \pi \lambda \tilde{\omega} \varsigma)$, pero hablaremos de nuevo más claramente en los [escritos] acerca de la poética-tercero ( $\tau \rho i ́ \tau o v)$, para

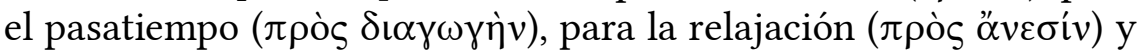

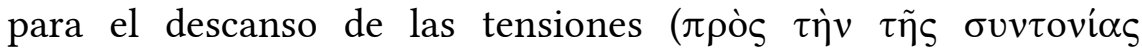

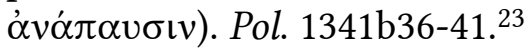

Como señalé al comienzo del trabajo, este pasaje plantea varias dificultades para el estudio de las funciones musicales. En primer lugar, resulta sorprendente que el filósofo mencione a la katharsis como una función diferenciada, a la cual no hizo referencia en el esquema de funciones propuesto en los capítulos previos. Además, parece identificar a la función más alta de la mousikēe esto es, aquella cuyo propósito es el pasatiempo, con la relajación y el descanso de las tensiones, que se emplea luego de un gran esfuerzo o trabajo duro. Aunque parezca una cuestión menor, esta aparente identificación contradice lo afirmado en los capítulos previos y no se condice con la importancia que Aristóteles le

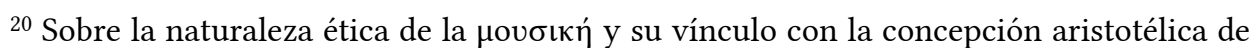
la virtud moral, cfr. Brüllman 2013.

${ }^{21}$ Kraut 1997, 193, quiere evitar toda posible identificación de ambas funciones y asegura que la referencia a las melodías de Olimpo solamente ejemplifica su influencia sobre el carácter, como un sustituto seguro para formas patológicas del entusiasmo, pero -a su juicio- ellas no prueban que la música puede hacer a los hombres virtuosos.

22 Mediante esta afirmación no propongo identificar la función catártica con la función ética, ya que el propio Aristóteles se encargará de distinguirlas en los capítulos siguientes. No obstante, el pasaje es significativo en la medida en que muestra la dificultad de precisar el lugar

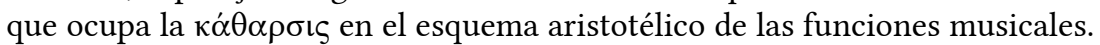

${ }^{23}$ Las traducciones de los pasajes de la Pol. propuestas a lo largo del trabajo son mías. 
otorga al ocio en su pensamiento ético-político. ${ }^{24}$ Respecto de la primera cuestión, podría argüirse que, en realidad, son cuatro los empleos de la mousikē que el filósofo aquí presenta, a saber, educativo, catártico, ocioso, destinado a la relajación y al descanso. ${ }^{25}$ Sin embargo, las reiteradas alusiones al número tres (incluso en este mismo pasaje) sugieren que el Estagirita adopta, al menos de manera expresa, un esquema tripartito. ${ }^{26}$

El propio Aristóteles reconoce que su análisis de las armonías y ritmos para la educación no es exacto y que ofrece un bosquejo general, el cual requiere de una consideración más detallada por parte de los músicos y los filósofos dedicados a la educación musical y también admite que ha tomado de otros filósofos la división de las melodías en éticas, prácticas y entusiásticas (Pol. 1341b23-34). ${ }^{27}$ En consecuencia, las imprecisiones y los problemas que plantea este pasaje de Pol. VIII 7 podrían, en cierta medida, atribuirse al carácter general y heredado del análisis propuesto. Incluso si aceptamos la propuesta de Destrée de considerar (2017) la referencia al ocio como una glosa, ${ }^{28}$ subsiste la pregunta si la katharsis constituye una función independiente, a la cual no hizo referencia en las enumeraciones previas o si, por el contrario, debe ser subsumida en alguna de las tres funciones inicialmente enunciadas. Hay quienes consideran que es "obvia" la identificación de la katharsis con la relajación. ${ }^{29}$ De hecho, existen algunos indicios en Pol. VIII 5-7 que abonan esta lectura, tal como la referencia al efecto tranquilizador ( $\kappa \propto \theta \iota \tau \alpha \mu \dot{v} v o u \varsigma)$ de los cantos sagrados y la comparación con su efecto curador y catártico (Pol. 1342a8-11). Además, la alusión al carácter báquico del aulós, entre los instrumentos, y del frigio, entre las armonías, los cuales paradigmáticamente representan lo orgiástico y lo patético (Pol. 1342b1-6), pareciera conectar a la katharsis con el juego y el descanso, ya que

${ }^{24}$ Como advierte Destrée 2017, 37, "lo que parece ser una pequeña objeción exegética o filológica realmente señala un asunto crucial, el núcleo mismo de la propuesta de Aristóteles, cuando Aristóteles describe la felicidad perfecta, que solo una bien concebida educación musical de los jóvenes hace posible". La traducción es de mi autoría.

${ }_{25}$ Al respecto, Kraut 1997, 209, sostiene que son cuatro los efectos musicales aquí enunciados y que es probable que los manuscritos no transmitan lo que Aristóteles originalmente escribió. En su último trabajo, Destrée 2017, 40, rechaza el esquema cuaternario que tácitamente adopta en un trabajo anterior (Destrée 2011,21) y sostiene que la referencia al

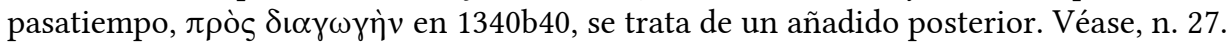
$1341 \mathrm{~b} 40$

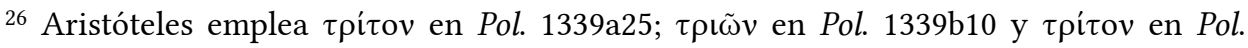

27 Teniendo en cuenta que Aristóteles adopta esta división no me parece correcto argüir, como propone Destrée 2017, 39-40, que la falta de correspondencia de esta con las funciones musicales, especialmente, en relación con las melodías que corresponderían al ocio, se deba a este carácter heredado, pues el propio Destrée reconoce que Aristóteles adopta esta división: "Aristóteles no está reestableciendo su propia división de los fines de la música y, por ende, no está agregando otro objetivo a aquellos que describió antes; él está informando una división que

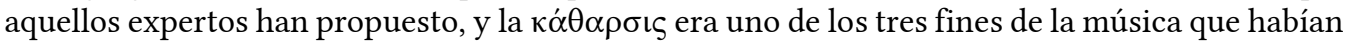
enumerado. Lo que es seguro es que Aristóteles respalda su división." [Las cursivas son mías]. La traducción es de mi autoría.

${ }^{28}$ Véase, n. 25.

${ }^{29}$ Cfr. Destrée 2011, 21; 2017, 39-40. 
en Pol. VIII 5 Aristóteles afirma que el sueño, la bebida, la mousikē y la danza tienen el mismo propósito: "hacen cesar las preocupaciones" (Pol. 1339a16-21). No hay dudas de que la relajación es uno de los efectos de la katharsis, tal como Aristóteles deja en claro en su descripción general del proceso que ponen en juego los cantos sagrados. Ahora bien, una de las dificultades que -a mi juicioenfrentan quienes establecen una identificación directa entre ambas es que, según esta lectura, la katharsis se correspondería con la función lúdica (siguiendo el esquema tripartito inicial), pero el proceso emocional que esta pone en juego y la relajación a la cual da lugar no parecieran ser de la misma clase que los que se producen a partir del empleo lúdico de la mousikē, esto es, el descanso de las tensiones del trabajo (Pol. 1339b15-17). Además, como vimos, es el propio Aristóteles, quien parece conectar ejemplarmente la katharsis con la formación del carácter mediante la alusión a las melodías de Olimpo, cuando describe la función ética de la mousikē. La evidencia más contundente que pone en tela de juicio la "obvia" identificación entre katharsis y relajación se encuentra en el pasaje antes mencionado de Pol. VIII 7, el cual claramente muestra que se trata de funciones diferenciadas, pues la katharsis no está incluida en la tercera función, ya sea que se acepte o no como auténtica la referencia al ocio: "no solo en virtud de la educación y de la katharsis [...] tercero ( $\tau \rho i ́ \tau o v)$, <para el pasatiempo $>$, para la relajación y para el descanso de las tensiones". ${ }^{30}$

A pesar de la novedad que conlleva el empleo de la noción de katharsis en Pol. VIII 7 respecto de sus consideraciones previas sobre las funciones de la mousike , cabe recordar que esta no es la primera aparición del término en el libro octavo, sino que Aristóteles ya hace alusión a él en Pol. VIII 6, a propósito de la prohibición del uso del aulós en la educación de los futuros ciudadanos. ${ }^{31}$ Allí no hace ninguna aclaración sobre el significado del término, simplemente asegura que este instrumento debe ser empleado cuando el espectáculo ( $\theta \varepsilon \omega \rho i \alpha)$ hace posible la katharsis más que el aprendizaje ( $\mu \alpha \dot{\theta} \theta \eta \sigma \iota v$ ) (Pol. 1341a21-24). Esta afirmación es muy significativa porque revela que la katharsis es el principal propósito de determinada clase de espectáculos, pero el empleo del comparativo ( $\mu \tilde{\alpha} \lambda \lambda \mathrm{ov}$ ) pone de manifiesto que tales espectáculos conllevan aprendizaje, si bien ese no es su principal objetivo. En consecuencia, pareciera ser que la katharsis y el aprendizaje no son -para Aristóteles- propósitos excluyentes de los espectáculos, lo cual quizás resulte valioso a la hora de contrastar el papel que le otorga a este término en Pol. y en Poet. ${ }^{32}$ Probablemente, la diferencia entre los distintos tipos de espectáculos resida en el grado en el que cada

${ }^{30}$ Mediante los ángulos, señalo lo que para Destrée constituye una glosa. Véase, n. 25.

${ }^{31}$ El aulós era un antiguo instrumento griego de viento, que todavía hoy suele ser confundido con la flauta. Aunque existían distintas clases, en el período clásico el más habitual era el de doble junco. Se caracterizaba por su capacidad para expresar y despertar emociones y era empleado para rituales religiosos. Cfr. West 1992, 1; 81-3; 93-6; 330-3; passim.

32 Cuestión de la que no me ocupo en el presente trabajo. 
uno propicia ora el aprendizaje ora el desborde emocional. No obstante, Aristóteles deja en claro que debido a su carácter orgiástico el aulós no puede formar parte de la educación, ya que impide el uso de la palabra (Pol. 1341a25) y, por ende, no contribuye en nada al entendimiento (Sióvoı $\alpha v$, Pol. 1341b6). Este pasaje podría considerarse como el primer antecedente de la escisión entre la formación ética y la katharsis musical que aparece explícitamente enunciada en el pasaje de Pol. VIII 7. En efecto, teniendo en cuenta las distintas consideraciones sobre la katharsis y, de manera general, sobre los instrumentos y las melodías vinculadas a ella que Aristóteles hace a lo largo de Pol. VIII 5-7, parece legítimo afirmar que se observa una progresiva separación entre ambas, desde la subsunción de las melodías de Olimpo en la función ética de la mousikē hasta la constitución de la katharsis como función independiente en el último capítulo de la obra.

En este punto de la exposición, es inevitable preguntar por el significado de la palabra katharsis o, más precisamente, qué es lo que Aristóteles entiende por este término en Pol. VIII. Su promesa incumplida de ocuparse del tema en los escritos sobre poética parece situarnos en un callejón sin salida, pues no está claro si está pensando en el libro II de la Poética o en su escrito esotérico Sobre los poetas o simplemente refiera a sus consideraciones generales sobre el tema (Destrée 2011, 25 n.1). Lo cierto es que no disponemos de ese o esos textos a los cuales remite y las dos únicas menciones del término en Poet. (119b27-28; 1455b15) no nos permiten esclarecer su sentido. Por otra parte, introducirse en el vasto e inagotable debate sobre el significado de este término en Aristóteles, el cual se inicia en el Renacimiento y pervive hasta nuestros días, resulta, en cierto modo, desalentador, a la vez que inevitable. En los últimos ciento cincuenta años, el debate ha virado desde las interpretaciones estrictamente médicas, las cuales estuvieron muy en boga desde mediados del siglo XIX, pasando por las lecturas de corte estético iniciadas en el ámbito anglosajón en los años sesenta y que tuvieron una fuerte influencia en la erudición francesa, hasta las

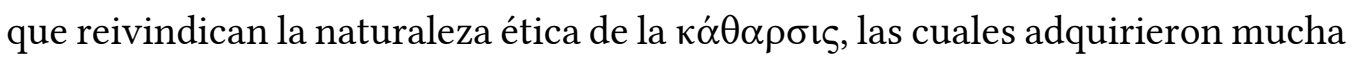
repercusión en los años noventa del siglo pasado. ${ }^{33}$

Más allá de los vaivenes e intereses filosófico-historiográficos que han intervenido en su recepción/apropiación, entiendo que en Pol. VIII 7 Aristóteles ofrece indicios muy significativos para comprender cómo entiende esta noción tan elusiva. Luego de la compleja presentación de las funciones musicales, afirma que deben emplearse todas las clases de armonías, pero aclara que cada una con distintos propósitos: "para la educación, las más éticas, mientras que para los que escuchan a otros ejecutando, las prácticas y las entusiásticas” (Pol. 1342a2-4). Esta triple división de las armonías en éticas, prácticas y entusiásticas

${ }^{33}$ Para un resumen de la historia de la apropiación de este concepto medular de la estética Occidental, cfr. Destrée 2011, 17 notas 1, 2 y 3; Halliwell 1986, 350-356. 
es muy problemática no solo por su carácter heredado sino, fundamentalmente, porque no está claro a qué función corresponden las melodías prácticas, en qué se diferencian de las éticas y cuál de las tres corresponde a la función más alta de la mousike esto es, la ociosa. ${ }^{34} \mathrm{El}$ análisis de estas cuestiones es tan complejo que ha impulsado a algunos editores e intérpretes a adoptar una enmienda textual que reemplaza $\tau \grave{\alpha} \kappa \alpha \theta \alpha \rho \tau \iota \kappa \grave{\alpha}$ por $\tau \grave{\alpha} \pi \rho \alpha \kappa \tau \tau \kappa \grave{\alpha}$ en Pol. 1342a15. ${ }^{35}$ A pesar de que la consideración de estas dificultades excede los límites del presente trabajo, no caben dudas respecto a que las armonías entusiásticas corresponden a la función catártica y son estas las que aquí nos interesan. De hecho, Aristóteles presenta inmediatamente a continuación, una extensa consideración sobre las emociones, que claramente corresponde a esta clase de melodías.

La emoción ( $\pi \dot{\alpha} \theta$ os) ocurre en algunas almas fuertemente, existe en todas, pero difiere en el menos y en el más, tal como la piedad y el miedo y, además, el entusiasmo ( $\dot{\varepsilon} v \theta 0 v \sigma \iota \alpha \sigma \mu$ ó $\varsigma)$. Pues, algunos son poseídos por este movimiento, a partir del efecto de los cantos sagrados, observamos que cuando estos emplean las melodías que ponen el alma fuera de sí son tranquilizados ( $\kappa(\theta \imath \sigma \tau \alpha \mu \dot{\varepsilon} v o v \varsigma)$, como ( sariamente, esto mismo padecen los piadosos y los miedosos y, en general, las personas emocionales ( $\pi \alpha \theta \eta \tau \iota \kappa o u ́ \varsigma)$, y los otros, en cuanto estas afecten a cada uno, y a todos les acaece cierta catarsis ( $\tau \iota v \alpha \kappa \alpha ́(\theta \alpha \rho \sigma \iota v)$ y alivio con placer. De igual modo, también las melodías catárticas/ <prácticas> producen goce inofensivo a los hombres. ${ }^{36}$ Pol. 1342a4-16

Este pasaje es fundamental para comprender el significado aristotélico de katharsis, pues es el único texto que disponemos en el que filósofo explica si bien de manera general $(\dot{\alpha} \pi \lambda \tilde{\omega} \varsigma)$ y mediante una comparación, qué es lo que ocurre durante la katharsis, a quiénes afecta y en qué medida. Sus consideraciones iniciales podrían sugerir que solo se refiere a quienes experimentan las emociones con mayor intensidad, en virtud de lo cual algunos intérpretes

${ }^{34}$ Destrée 2017, 37-38, ofrece una breve reseña de las numerosas enmiendas que han sido propuestas en relación a este pasaje, desde fines del siglo XIX y principios del XX por los editores y comentadores ingleses y alemanes.

35 En su edición de 1957, Ross recoge la enmienda propuesta por Sauppe, la cual es adoptada por algunos intérpretes, si bien hay quienes se ajustan al texto conservado en los manuscritos. La decisión interpretativa de adoptar o no esta enmienda es decisiva para explicar cuáles melodías corresponden a la función ociosa. Así, por ejemplo, Kraut 1997, 208, 48 ad loc., quien se atiene al texto original, vincula las melodías prácticas -entendidas en términos de acción- al ocio. Por su parte, Destrée 2013, 318 n. 19; 2018, acepta la enmienda y postula una correspondencia fáctica entre las melodías éticas y aquellas destinadas al ocio, ya que -a su juicio- las prácticas no están vinculadas a la acción, sino al movimiento y solo se emplearían para la relajación luego del trabajo duro.

${ }^{36}$ Sobre esta enmienda textual, véase la nota anterior. 
entienden que solo refiere a estos (Belfiore 1992, 326), los cuales podrían denominarse enfermos emocionales. Sin dudas, resulta significativo que Aristóteles ilustre el proceso emocional que la katharsis pone en juego mediante su empleo cultual, aun cuando su análisis de la mousikē en Pol. VIII se centra en los espectáculos teatrales y no en su uso propiamente religioso. El ejemplo de los cantos sagrados revela que la katharsis produce, en primer lugar, un estado de éxtasis, un salirse de sí mismo que se logra mediante determinada clase de melodías (como sería el caso de las melodías de Olimpo, a las cuales refirió en Pol. VIII 5), y, después del cual, el individuo es tranquilizado. Este proceso de expurgación emocional es similar a una curación y a una purgación médica, gracias al cual se logra la calma y se reestablece el equilibrio. Justamente, el empleo del comparativo $̋ \sigma \pi \varepsilon \rho$ (como si ocurriera curación y katharsis) y del indefinido $\tau \iota v \alpha$ (cierta katharsis) revela que Aristóteles no emplea literalmente el término en sentido médico, sino solo de manera ilustrativa. ${ }^{37} \mathrm{El}$ programa pedagógico que diseña para el mejor régimen y, en particular, el currículo musical que bosqueja en Pol. VIII 3, 5-7 apuntan a fomentar la moderación emocional, la cual es indispensable para el logro de la virtud de los futuros ciudadanos. Por ese motivo, el Estagirita establece estrictas restricciones sobre todo aquello que provoque lo extático, el estar fuera de sí; en definitiva, el descontrol emocional. De allí que, como vimos, prohíba que los futuros ciudadanos ejecuten el aulós o cualquier instrumento que requiera destreza técnica (Pol. 1341a19-21) y tampoco permite que las armonías frigias intervengan en su educación (Pol. 1340b4-5; 1342a32-b6). A partir de lo cual, podría inferirse que los ciudadanos del mejor régimen no pueden participar de ningún espectáculo o actividad que involucre la katharsis, la cual solo estaría limitada a la clase de los espectadores vulgares, conformada por trabajadores manuales, asalariados y otros de esta clase (Pol. 1342a19-20). Retomando la idea aristotélica conforme a la cual no hay ocio y, por ende, felicidad, para los que trabajan (Pol. 1338a1-3), análogamente podría pensarse que no habría katharsis para los ciudadanos del mejor régimen. Sin embargo, Aristóteles aclara que las emociones afectan a todos, diferenciándose solo en el grado con el que las experimentan; además, asegura que el proceso emocional que involucra la katharsis es el mismo en todos los casos. ${ }^{38}$ Más aún, el Estagirita propone una clasificación de los hombres según su grado de respuesta emocional, la cual se corresponde con una experiencia catártica de cierta

${ }^{37}$ Aristóteles recurre a la comparación con las curas médicas en Pol. 1339 b17-17 a pro-

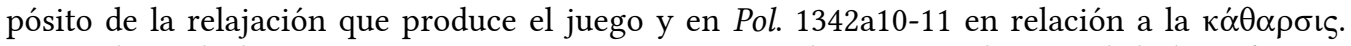

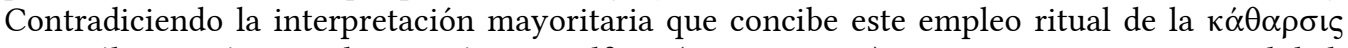
aristotélica en términos homeopáticos, Belfiore (1992, 324-325) sostiene que esta es, en realidad, de naturaleza alopática, lo cual se condice -a su juicio- con el principio de los opuestos que se aplica a los tratamientos médicos y que está presente en su concepción física y psíquica de la

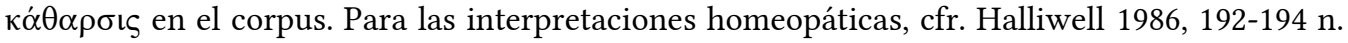
37, Destrée 2011, 34-35.

38 Sobre esta cuestión me ocuparé con más detenimiento más adelante en el trabajo. 
clase $^{39}$. En efecto, en primer lugar, refiere al caso emocionalmente extremo de quienes padecen el entusiasmo y cuya respuesta emocional se corresponde con la katharsis ritual. Justamente, Aristóteles apela a esta forma extrema como ejemplo para explicar el proceso que, de manera general, la katharsis pone en juego. Tal como he señalado anteriormente, el uso del comparativo impide identificarla con la cura y la catarsis en términos médicos; sin embargo, el empleo de este vocabulario sugiere que se trata de un tipo de experiencia emocional análogo a una enfermedad. Asimismo, distingue otras dos clases de personas, a saber, quienes viven con intensidad ciertas emociones, como es el caso paradigmático del piadoso y del miedoso y, en general, de los hombres emocionales. Por último, parece incluir a un espectro más grande de individuos (los otros, Pol. 1342a13) que caerían en un rango más moderado de respuesta emocional. El hecho de que afirme que en todos ( $\pi \tilde{\alpha} \sigma \mathrm{l}$, Pol. 1342a14) se produce cierta catarsis y alivio con placer, revela que el proceso involucrado en los tres casos es el mismo. ${ }^{40}$ Sin embargo, es sugestivo que a esta segunda aparición del término la califique con un pronombre indefinido, mientras que a la primera la vincule con la cura, lo cual pone de manifiesto que la última categoría, implica un proceso emocional menos intenso que su utilización ritual casi patológica. En definitiva, todas las emociones humanas, independientemente de su intensidad y de la posición que el individuo ocupe en el régimen político, conllevan como respuesta una cierta forma de katharsis, lo que varía es el grado en que esta se da en cada caso.

Si bien Aristóteles no especifica a cuál de estas tres categorías de personas deben pertenecer los ciudadanos del mejor régimen, es muy probable que considerara que estos podían experimentar emociones intensas y, por ende, pudieran participar de espectáculos o incluso ceremonias religiosas que propiciaran cierta forma de katharsis ritual. Tal vez, por ese motivo el filósofo limita las armonías entusiásticas (junto a las prácticas) a quienes escuchan lo que ejecutan otros, dejando abierta la posibilidad de que los ciudadanos puedan formar parte del auditorio de esa clase de armonías, a la vez que impide que sean sus ejecutantes, lo cual se condice con sus prescripciones sobre la ejecución musical. ${ }^{41}$ En tal sentido, cabe recordar que en sus consideraciones sobre la censura en Pol. VII 17 reconoce que los ciudadanos pueden contemplar representaciones inde-

${ }^{39}$ Cfr. Halliwell 1986, 191 y 194 y Kraut 1997, 210-211.

${ }^{40}$ Por el contrario, para Belfiore (1992, 326), mediante este empleo de $\pi \tilde{\alpha} \sigma \mathrm{l}$ (Pol. 1342a14), Aristóteles no refiere a todos los hombres, sino a quienes padecen excesivamente de las emociones. Para lecturas más comprensivas, cfr. Lord 1982, 130-134, Destrée 2011, 21-22 y Halliwell 1986, 191.

${ }^{41}$ Ford 2004, 329, interpreta este argumento en sentido completamente contrario, pues asegura que si las melodías catárticas (y prácticas) tuvieran valor educacional, deberían ser

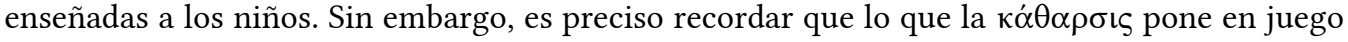
no es el discernimiento ético o estético, el cual requiere de una preparación previa, sino un proceso emocional, gracias al cual se alivian las emociones intensas y se recupera el equilibrio. 
centes en los templos de cierta clase de dioses e incluso participar de espectáculos de yambos y comedias una vez alcanzada la edad en la que pueden participar de las comidas en común y beber, porque -asegura- solo entonces la educación los habrá hecho indemnes ( $\alpha \pi \alpha \theta \varepsilon \tilde{\iota} \varsigma)$ al daño resultante de tales cosas (Pol. 1336b14-23). A la luz de ello, es posible suponer que los ciudadanos del mejor régimen pueden eventualmente ser espectadores o participar de cultos que provoquen una experiencia catártica intensa, pero solo una vez que han alcanzado la moderación y el dominio de sus emociones y han sido habituados musicalmente en la virtud, es decir, únicamente cuando la educación musical los ha hecho indemnes al daño que para el ciudadano supone el estar fuera de sí. ${ }^{42}$ En definitiva, las emociones y, en particular, los desbordes emocionales -ya sea en ámbitos artísticos y/o cultuales- son parte de la vida de todos los hombres, incluso de los ciudadanos que han alcanzado la moderación y son capaces de disfrutar del ocio musical. Precisamente, en este sentido es legítimo afirmar que la función ética de la mousikē es condición de la catártica.

3

Más allá de si se acepta que son tres o cuatro las funciones de la mousikē, el lugar que ocupa la katharsis en el esquema de las funciones musicales es incómodo, pues no resulta convincente la propuesta de subsumirla en la función lúdica ni en la educativa ni tampoco la de afirmar con certeza su independencia. Aunque su efecto relajante y la comparación con la cura médica sugieren una conexión con la primera, la relajación que produce y el placer que conlleva no son de la misma clase que la relajación y el placer que se experimenta después de un trabajo duro. A lo largo del artículo, hemos visto que la identificación de la katharsis con la relajación no resulta tan obvia como algunos autores afirman, lo cual se pone en evidencia en el complejo pasaje de Pol. VIII $7 .{ }^{43} \mathrm{El}$ hecho de que Aristóteles apele a las melodías de Olimpo, que tienen un efecto extático, para ejemplificar la influencia ética de la mousikē en el carácter sugiere su vínculo con la función educativa. A lo cual, podría añadirse que el filósofo no establece una contraposición absoluta, sino comparativa entre los espectáculos que tienen como propósito la katharsis y aquellos que apuntan al aprendizaje. Sin embargo, también deja en claro que la katharsis no educa moralmente a los futuros ciudadanos. Si bien todas las experiencias emocionales involucran una cierta clase de katharsis, es probable que sus formas más extremas solo estén permitidas durante la adultez, cuando estos ya han adquirido la virtud. El descontrol emocional y la posterior relajación que, de manera general, la katharsis produce, no forman parte del proceso de habituación a la virtud, pero lo presuponen. Además, hemos visto que la relación entre katharsis y educación

\footnotetext{
${ }^{42}$ Cfr. Kraut 1997, 209; Destrée 2011, 22.

43 Cfr. Destrée 2011, 21; Veloso 2007, 262.
} 
ni siquiera es uniforme a lo largo de los tres capítulos en los que tácita o explícitamente refiere a ella, tal como demuestra la progresiva separación que se observa desde su unidad en Pol. VIII 5, su incipiente separación en Pol. VIII 6 y la constitución de la katharsis como función independiente de la mousikē en Pol. VIII 7. De hecho, esta última parece una explicación factible, pero se choca con la insistente afirmación aristotélica de que son tres las funciones musicales.

A la luz de todo lo expuesto, podría decirse que la katharsis está en una posición oscilante entre la relajación y la educación. De hecho, hay lecturas del texto que postulan una unidad entre la relajación y la purgación, mientras que otras reconocen una unidad genérica formada por la educación moral y la katharsis. ${ }^{44}$ Aunque resulte desalentador admitirlo, quizás no sea posible ofrecer una respuesta taxativa a esta cuestión. Pero lo que esta dificultad pone en evidencia es la riqueza que la consideración general sobre la katharsis que ofrece en Pol. VIII 7 tiene para comprender los distintos propósitos de la mousikē en el programa educativo aristotélico y no solo como mero instrumento para elucidar su papel en la definición de tragedia en Poet. 6. En tal sentido, entiendo que es preciso purgarnos - si acaso, ello es posible- de la vasta historia de las interpretaciones que se han dado de la katharsis musical con vistas a esclarecer su significado en Poética, para comenzar a entender la singularidad y la riqueza de su empleo en el último libro de la Política, comprendiéndola como una parte necesaria del currículo musical el cual, a su vez, integra el programa educativo del mejor régimen. A pesar de las dificultades que -como he indicado a lo largo del trabajo- implica dicho estudio, resulta indispensable para esclarecer el papel que ocupan las emociones en el proyecto político de Aristóteles.

\section{BIBLIOGRAFIA}

Belfiore, E. 1992. Tragic Pleasures: Aristotle on Plot and Emotion. Princeton: Princeton University Press.

Brüllmann, Ph. 2013. "Music Builds Character Aristotle, Politics VIII 5, 1340a14b5”. Apeiron 46 (4): 345-373.

Destrée, P. 2011. "La purgation des interprétations: conditions et enjeux de la catharsis poétique chez Aristote". En Littérature et thérapeutique des passions: La catharsis en question, editado por Jean-Charles Darmon, 1435. Paris: Hermann.

44 Destrée 2017, 38. defiende la primera lectura frente a la unidad genérica entre

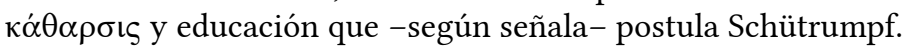


Destrée, P. 2013. "Education, leisure, and politics". En The Cambridge Companion to Aristotle's Politics, editado por Marguerite Deslauriers y Pierre Destrée, 301-323. Cambridge: Cambridge University Press.

Destrée, P. 2017. "Aristotle and Musicologists on Three Functions of Music. A note on Pol. 8, 1341b401", Greek and Roman Musical Studies 5: 35-42.

Destrée, P. 2018. "Aristotle on Music for Leisure". En Music, Texts, and Culture in Ancient Greece, editado por Armand D'Angour y Thomas Philips, 183202. Oxford: Oxford University Press.

Ford, A. 2004. "Catharsis: The Power of Music in Aristotle's Politics". En Music and the Muses, editado por Penelope Murray y Peter Wilson, 309-336. Oxford: Oxford University Press.

Halliwell, S. 1986. Aristotle's Poetics. Chicago: University of Chicago Press.

Kraut, R. 1997. Aristotle Politics Books VII and VIII. Oxford: Oxford University Press.

Livov, G. 2015. Aristóteles. Política. Traducción, estudio preliminar y notas. Bernal: UNLQ/Prometeo.

Lord, C. 1982. Education and Culture in the Political Thought of Aristotle. New York: Cornell University Press.

Nightingale, A. 1996. "Aristotle on the 'Liberal' and 'Illiberal' Arts", Proceedings of the Boston Area Colloquium of Ancient Philosophy 12 (1): 29-58.

Rocconi, E. 2010. "Sounds of war, sounds of peace: for an ethnographic survey of ancient Greek music in Platonic writings". En Musical Perceptions - Past and Present. On Etnographic Analogy in Music Archeology, editado por Ricardo Eichmann, Ellen. Hickmann y Lars Christian Koch, 117-129, Rahden: Leidorf.

Rocconi, E. 2015. "Music and Dance in Greece and Rome". En A Companion to Ancient Aesthetics, editado por Pierre Destrée y Penelope Murray, 81-93. Oxford: Wiley-Blackwell.

Ross, W. D. 1957. Aristotle's Politics. Oxford: Clarendon Press.

Santa Cruz, M. I. - Crespo, M. I. 2005. Aristóteles. Política. Introducción, traducción y notas. Buenos Aires: Losada.

Suñol, V. 2015. "La educación como fundamento del (mejor) régimen político en Aristóteles”, Éndoxa: Series Filosóficas 36 (2): 56-73.

Suñol, V. 2017. "La educación musical en Aristóteles: su correspondencia con la vida mejor en el mejor régimen", Boletín de Estética XIII (41): 8-37.

Veloso, C. W. 2007. “Aristotle's Poetics without Katharsis, Fear, or Pity”. En Oxford Studies in Ancient Philosophy, editado por David Sedley, 255-284. Oxford: Oxford University Press.

West, M. 1992. Ancient Greek Music. Oxford: Oxford University Press. 